\title{
CHARTING COOL CARBON STARS IN THE LARGE MAGELLANIC CLOUD
}

\author{
M.F. McCARTHY, S.J. \\ Vatican Observatory Research Group \\ Steward Observatory \\ University of Arizona \\ Tucson AZ 85721 \\ USA \\ V.M. BLANCO \\ Cerro Tololo Inter-American Observatory \\ Casilla 603 \\ La Serena \\ Chile
}

\begin{abstract}
We describe the preparation of charts and positions for 1035 cool red giant carbon stars discovered by the authors in the Large Magellanic Cloud (LMC). Not considered here are Small Magellanic Cloud (SMC) carbon stars, nor the M-type giants found in the LMC and SMC (Blanco et al. 1980, Blanco \& McCarthy 1983). Charts of the C stars discovered are available in three formats: (a) 12 charts contain $186 \mathrm{C}$ stars discovered in three central LMC regions (Blanco et al. 1980); (b) charts of C and late $\mathrm{M}$ stars in $52 \mathrm{LMC}$ regions; these charts will not be published but are available from the authors (Blanco \& McCarthy 1983, discussion); and (c) charts for 849 individual C stars in 49 regions of LMC (Blanco \& McCarthy 1990).
\end{abstract}

To discover cool red giant stars in the LMC, we used the Curtis Schmidt, the $4 \mathrm{~m}$ and $1.5 \mathrm{~m}$ reflectors, all at CTIO. Schmidt work with a thin prism showed a mean $\mathrm{m}_{i}=14.0$ for late type stars in the LMC but the dispersion $(6300 \AA / \mathrm{mm})$ was judged to be too low for the proper separation of $C$ from late type $M$ stars. When the $4 \mathrm{~m}$ CTIO reflector was introduced we installed a grism at the prime focus and were able to reach a limiting magnitude near $\mathrm{m}_{i}=18.0$. Grism dispersion was $2350 \AA / \mathrm{mm}$ and the $C$ stars could be readily distinguished from the late $M$ type stars. Kodak I N and IV N plates, hypersensitized with $\mathrm{Ag} \mathrm{NO}_{3}$ were exposed through a Schott 695 filter for the grism work; the direct plates used for charting and positional work were Kodak 098 red sensitive plates exposed through a Schott RG 610 filter. Our photographic observing programs were ending just as the days of the CCD were dawning.

Accurate positions for finding faint C stars in the three central fields of LMC (1980) were obtained by measures using the Vatican Zeiss 2-coordinate machine. For the outer 49 regions of the LMC the authors (1990) employed non-photometric nights on the $1.5 \mathrm{~m}$ telescope to make offsets to measure the positions of C stars; SAO stars served as references; further complications arose from the crowded fields and the lack of sufficient standard stars in the small grism fields. Concerned to share with colleagues the results of our $\mathrm{C}$ star discoveries, we chart the $\mathrm{C}$ stars and list finding positions. Three different procedures for presentation of our results were followed; of these, two are published and one is not .

The first survey concerned $186 \mathrm{C}$ stars in three central regions of the LMC and for these the authors (1980) published : finding charts, positions, magnitudes and (R-I) colours. Each circular 
grism region was divided into four quadrants; the scale as published was $6.21 / \mathrm{mm} ; 186 \mathrm{C}$ stars were marked in the three regions called the Optical Centre, the Radio Centre and the Bar West Region.

The second and third presentations of data extend the survey to 49 external regions. From counts of C stars, isopleths of surface distribution of C stars in the LMC were constructed. A discussion of these results was published by the authors (1983).

In the second presentation the $C$ stars as well as the late type $M$ giant stars were mapped. We found that the cost of publication for these 49 regions on the scale of our earlier work on the central regions would have been excessive. So these charts will not be published; colleagues who wish to consult them may write to one of the authors. The original scale of these was $11.11 / \mathrm{mm}$.

The third method presents, for each $\mathrm{C}$ star discovered in the 49 regions, an individual chart similar to the finding charts customarily published for variable star discoveries. These charts have been accepted for publication by the Astronomical Journal and, as before, the C stars are included between two bars. Occasionally, where two or three $\mathrm{C}$ stars are found to exist in close vicinity, all are marked on the same chart. Each field chart measures $115 \operatorname{arcsec}$ by 115 arcsec. In our publication there are ten pages of $C$ stars; the total number of stars shown is 849 . Separate pages list the centres for all 49 regions in LMC. Then, the C stars discovered are given, with the approximate finding position. The accuracy of the published positions depends on the frequency of available standard stars. In the least favourable cases this is $12 \operatorname{arcsec}$ and in most cases amounts to 4 arcsec. The experience of the authors and of colleagues who have used our data is that these positions, when used with the charts, are sufficient for finding the $\mathrm{C}$ stars discovered. Astrometric accuracy was limited by the telescope time available. So, precise astrometry, photometry and colorimetry must be a project for the future, together with higher dispersion slit spectra of the $\mathrm{C}$ stars reported here.

Many interesting features in analyzing the role of the $\mathrm{C}$ stars in the LMC remain for future work. Probably, all of the $\mathrm{C}$ stars are at least slightly variable. Problems connected with the various subclasses among the Carbon stars will merit further investigation. The kinematics of these stars in the LMC can be compared with similar studies now in progress in our own galaxy. The results reported here can supply, if not the "giant's shoulders" for these tasks, then they may, at least, furnish to colleagues a "helping hand".

\section{References}

Blanco, V.M., McCarthy, S.J., M.F. and Blanco, B.M. (1980), Astrophys. J. 242, 938.

Blanco, V.M., McCarthy S.J., M.G. (1983), Astron. J. 88, 1442.

Blanco, V.M., McCarthy S.J., M.F. (1990), Astron. J. 100, 674, 977. 